\title{
Determinants of in-hospital and long-term surgical outcomes after repair of postinfarction ventricular septal rupture
}

\author{
Satsuki Fukushima, MD, PhD, ${ }^{\mathrm{a}}$ Peter J. Tesar, FRACS, ${ }^{\mathrm{a}}$ Homayoun Jalali, FRACS, ${ }^{\mathrm{a}}$ \\ Andrew J. Clarke, FRACS, ${ }^{\mathrm{a}}$ Hemant Sharma, MCh, ${ }^{\mathrm{a}}$ Jivesh Choudhary, MCh, ${ }^{\mathrm{a}}$ Harry Bartlett, PhD, ${ }^{\mathrm{b}}$ and \\ Peter G. Pohlner, FRACS ${ }^{\mathrm{a}}$
}

\begin{abstract}
Objectives: Surgical repair of post-myocardial infarction ventricular septal rupture is challenging with reported early mortality being substantial. In addition, congestive cardiac failure and ventricular tachyarrhythmia frequently occur long term after the operation, although frequency and predictive factors of these events have been poorly identified.
\end{abstract}

\begin{abstract}
Methods: A consecutive series of 68 patients who underwent repair of postinfarction ventricular septal rupture by 14 surgeons between 1988 and 2007 was studied. Fifty-eight (85\%) patients underwent repair in an urgent setting ( $<48$ hours after diagnosis). Coronary artery bypass grafting was concomitantly performed in $48(71 \%)$ patients Mean follow-up period was $9.2 \pm 4.9$ years.

Results: Thirty-day mortality was $35 \%$, with previous myocardial infarction, previous cardiac surgery, preoperative left ventricular ejection fraction less than $40 \%$, and urgent surgery being independent risk factors. Actuarial survival of 30-day survivors was $88 \%$ at 5 years, $73 \%$ at 10 years, and $51 \%$ at 15 years. Actuarial freedom from congestive cardiac failure and ventricular tachyarrhythmia was $70 \%$ and $85 \%$ at 5 years, $54 \%$ and $71 \%$ at 10 years, and $28 \%$ and $61 \%$ at 15 years, respectively. Independent predictors for congestive cardiac failure included hypertension, posterior septal rupture, residual interventricular communication, and preoperative left ventricular ejection fraction less than $40 \%$, whereas concomitant ventricular aneurysmectomy and preoperative occlusion of the left anterior descending artery were independent predictors of ventricular tachyarrhythmia.
\end{abstract}

Conclusions: Long-term outcomes after surgical repair of postinfarction ventricular septal rupture was favorable, despite infrequent exposure by individual surgeons to the pathologic features, indicating that an aggressive surgical approach is warranted. Predictors of congestive cardiac failure and ventricular arrhythmia long term varied. (J Thorac Cardiovasc Surg 2010;140:59-65)

Ventricular septal rupture (VSR) after myocardial infarction (MI) is an infrequent condition with extremely high mortality, inasmuch as significant VSR eventually leads to congestive cardiac failure and cardiogenic shock without treatment. ${ }^{1-3}$ Current guidelines of the American College of Cardiology and the American Heart Association recommend immediate operative intervention in patients with postinfarction VSR regardless of their clinical status. ${ }^{4}$ Nonetheless, surgical repair of VSR is still challenging, with reported in-hospital mortality being substantial $(20 \%-$ $60 \%)^{2,5-7}$ Preoperative hemodynamic instability, posterior septal rupture, and incomplete revascularization have been

\footnotetext{
From the Department of Cardiothoracic Surgery, ${ }^{a}$ The Prince Charles Hospital, Chermside, and the School of Mathematical Sciences, ${ }^{\mathrm{b}}$ Queensland University of Technology, Brisbane, Australia. Disclosures: None.

Received for publication May 20, 2009; revisions received Aug 13, 2009; accepted for publication Sept 7, 2009; available ahead of print Nov 18, 2009.

Address for reprints: Satsuki Fukushima, MD, PhD, Department of Cardiothoracic Surgery, The Prince Charles Hospital, Rode Road, Chermside, QLD 4032, Australia (E-mail: Satsuki_Fukushima@health.qld.gov.au). 0022-5223/\$36.00

Crown Copyright (c) 2010 Published by Elsevier Inc. on behalf of The American Association for Thoracic Surgery

doi:10.1016/j.jtcvs.2009.09.018
}

suggested as risk factors for high in-hospital mortality after repair, although they are still inconsistent. ${ }^{3,6-15}$

Although long-term survival after repair of postinfarction VSR has been reported to be favorable, congestive cardiac failure and ventricular tachyarrhythmia are known to frequently impair quality of life long term. . $^{3,6,7,9,11,12,16,17}$ Transmural MI generating a VSR affects ventricular function and has the capacity to generate a macro-reentry circuit, inducing congestive cardiac failure and ventricular tachyarrhythmia, respectively. In addition, repair of ruptured septum with excision or exclusion of the infarcted myocardium might also alter ventricular geometry to adversely affect long-term ventricular function and electrical stability. Importantly, predictive factors of these events are poorly understood.

The goals of this study were to investigate in-hospital and long-term outcomes after VSR repair and to identify factors affecting congestive cardiac failure and ventricular tachyarrhythmia long term.

\section{METHODS}

A consecutive series of all patients who underwent surgical repair of postinfarction VSR in The Prince Charles Hospital between 1988 and 2007 was enrolled. The prospective departmental database identified that 


$$
\begin{aligned}
& \text { Abbreviations and Acronyms } \\
& \text { CABG }=\text { coronary artery bypass grafting } \\
& \text { LAD }=\text { left anterior descending coronary artery } \\
& \text { LV } \quad=\text { left ventricular } \\
& \text { LVEF }=\text { left ventricular ejection fraction } \\
& \text { MACE }=\text { main adverse coronary event } \\
& \text { MI } \quad \text { myocardial infarction } \\
& \text { VSR }=\text { ventricular septal rupture }
\end{aligned}
$$

$68(0.18 \%)$ patients among a total of 37,177 undergoing cardiac surgery were operated on by 14 individual surgeons during this study period. Additional information was obtained from medical records and interviews. This study was approved by the institutional ethics committee (Ref.; EC2893). Management strategy for VSR has been consistently (1) urgent preoperative angiography and (2) subsequent urgent or semiurgent VSR repair with complete revascularization over the study period

\section{Diagnosis and Preoperative Management for Postinfarction VSR}

Coronary angiography was preoperatively performed in $65(96 \%)$ patients, whereas 2 patients with cardiogenic shock and 1 patient with impaired renal function underwent urgent VSR repair without coronary angiography. Of 65 patients who underwent coronary angiography preoperatively, $20(31 \%)$ patients had a single diseased major coronary artery, whereas $45(69 \%)$ patients had multiple coronary artery disease. The number of diseased coronary arteries was not significantly different over the study period. Percutaneous coronary intervention was preoperatively performed in 3 patients $(4 \%)$.

\section{Surgical Strategy for Postinfarction VSR}

Fifty-eight $(85 \%)$ patients underwent surgery in an urgent setting $(<48$ hours after diagnosis of VSR), whereas $10(15 \%)$ patients underwent surgery in an elective setting inasmuch as the diagnosis was made incidentally without hemodynamic instability. All the operations were carried out with the aid of cardiopulmonary bypass with moderate hypothermia and cardiac arrest induced by infusing cardioplegic solutions antegradely and retrogradely every 20 minutes. Cold crystalloid predominated as the cardioplegic solution in the initial 10 years, whereas cold oxygenated blood predominated in the subsequent 10 years. Approaches to the ruptured septum were left ventriculotomy in $48(71 \%)$ patients, right ventriculotomy in $12(18 \%)$ patients, and both ventriculotomies in $6(9 \%)$ patients, left atriotomy with mitral valve annulus being detached and reattached in 1 patient and right atriotomy through tricuspid valve in $1 \mathrm{pa}-$ tient, depending on the infarct area. A patch was placed to repair the ruptured septum in $67(99 \%)$ patients, using artificial material in $50(74 \%)$, bovine pericardium in $15(22 \%)$, and autologous pericardium in $2(3 \%)$, depending on the size and shape of the VSR. Infarctectomy with septal reconstruction ${ }^{18}$ predominated in the initial 5 years, whereas minimal infarctectomy with a patch placement to exclude infracted, ruptured septum ${ }^{19}$ predominated in the subsequent 15 years.

Coronary artery bypass grafting $(\mathrm{CABG})$ was concomitantly performed in $48(71 \%)$ patients with an average number of bypass grafts being 1.7. A side branch of the culprit coronary artery for infarct VSR was revascularized in $22(32 \%)$ patients. In 15 patients, significantly diseased coronary arteries in the remote area from the culprit artery were not revascularized owing to ungraftable quality and/or size, defined as incomplete revascularization. The left internal thoracic artery was grafted to the left anterior descending artery (LAD) in 3 patients who were hemodynamically stable and had severe multiple coronary artery disease, whereas saphenous veins were used in the other grafts. Concomitant left ventricular (LV) aneurysmectomy was performed in $4(6 \%)$ patients having aneurysmal formation in the LV apex. Concomitant mitral valve surgery was performed in 1 patient having moderate mitral regurgitation. A bilateral ventricular assist device was implanted for global myocardial dysfunction in 1 patient. All patients were postoperatively examined by echocardiography in the intensive care unit.

\section{Statistical Analysis}

Continuous variables are presented as mean \pm standard deviation and/or median (interquartile range). Categorical variables are shown as the percentage of the sample. Comparison between 30-day survivors and nonsurvivors was performed by the Student $t$ test, $\chi^{2}$ test, or Fisher exact test as appropriate. Independent risk factors of 30-day mortality were identified by multivariate logistic regression analysis. The potential predictors entered to logistic regression are those showing $P<.100$ at the univariate analysis (Table 1). Actuarial survival, freedom from main adverse coronary events (MACE), freedom from congestive cardiac failure, and freedom from ventricular tachyarrhythmia were estimated by the Kaplan-Meier method. Independent predictive factors for congestive cardiac failure and ventricular arrhythmia were identified by the Cox proportional hazard model. The potential predictors entered to the Cox proportional hazard model are those that showed $P<.500$ in an initial proportional hazard model including the following predictors: year of surgery, 70 years of age or more at surgery, female gender, hypertension, diabetes, previous MI, preoperative serum creatinine greater than $250 \mu \mathrm{mol} / \mathrm{L}$, MI onset until surgery less than 48 hours, posterior septal rupture, preoperative LV ejection fraction (LVEF) less than $40 \%$, 3-vessel disease, occlusion of the LAD, incomplete revascularization, not-revascularized culprit artery, concomitant aneurysmectomy, and residual interventricular communication. Statistical analysis was performed using StatView-J 5.0 (SAS Institute, Inc, Cary, NC).

\section{RESULTS \\ Thirty-Day Mortality and Morbidities}

Among a total of 68 patients, $24(35 \%)$ deaths occurred within 30 days after repair of postinfarction VSR at a median of 3.5 postoperative days ( $0-27$ days). Five ( $7 \%$ ) patients died in the operating room owing to global myocardial dysfunction. Causes of the deaths in the patients who were successfully transferred to the intensive care unit after the operation were persistent low cardiac output in 13 patients, sepsis in 2 patients, ventricular arrhythmia in 1 patient, uncontrollable bleeding from a ventricle in 1 patient, and anemia in 2 patients who rejected blood transfusion on religious grounds. Six $(10 \%)$ patients underwent re-exploration for postoperative bleeding. Three $(5 \%)$ patients had permanent neurologic deficits, and $3(5 \%)$ had transient neurologic disorders. Hemodialysis was carried out for acute renal failure in $12(19 \%)$ patients. Of them, 7 patients died within 30 days postoperatively, whereas 5 patients survived and did not require further hemodialysis. Four patients underwent intervention of residual interventricular communication within 30 days postoperatively.

\section{Risk Factors for 30-Day Mortality}

Comparison between 30-day survivors and nonsurvivors was carried out in a univariate manner (Table 1). Of 5 patients who had previous cardiac surgery, only 1 patient 
TABLE 1. Thirty-day survivors versus nonsurvivors

\begin{tabular}{lccc}
\hline & $\begin{array}{c}\text { Survivors } \\
(\mathbf{n}=\mathbf{4 4})\end{array}$ & $\begin{array}{c}\text { Nonsurvivors } \\
(\mathbf{n}=\mathbf{2 4})\end{array}$ & $\begin{array}{c}\boldsymbol{P} \\
\text { value }\end{array}$ \\
\hline Background & & & \\
Year of surgery & & & .387 \\
$\quad$ 1988-1992 & $20(63 \%)$ & $12(38 \%)$ & \\
$1993-1997$ & $13(81 \%)$ & $3(19 \%)$ & \\
1998-2002 & $5(50 \%)$ & $5(50 \%)$ & \\
2003-2007 & $6(69 \%)$ & $4(40 \%)$ & \\
Age & $66 \pm 10$ & $67 \pm 8$ & .698 \\
Female gender & $14(32 \%)$ & $5(21 \%)$ & .335 \\
Hypertension & $28(64 \%$ & $13(54 \%)$ & .446 \\
Diabetes & $7(16 \%)$ & $6(25 \%)$ & .520 \\
Previous MI & $7(16 \%)$ & $8(33 \%)$ & .098 \\
Previous cardiac surgery & $1(2 \%)$ & $4(17 \%)$ & .049 \\
Preoperative condition and & & & \\
$\quad$ pathology & & & \\
Heart rate (beats/min) & $103 \pm 17$ & $109 \pm 17$ & .194 \\
Systolic BP $<100$ mm Hg & $16(36 \%)$ & $21(88 \%)$ & $<.0001$ \\
Serum creatinine $>$ 250 $\mu$ mol/L & $5(11 \%)$ & $8(33 \%)$ & .050 \\
Inotrope support & $12(27 \%)$ & $13(54 \%)$ & .028 \\
IABP support & $10(23 \%)$ & $18(75 \%)$ & $<.0001$ \\
Mechanical ventilation & $2(5 \%)$ & $3(13 \%)$ & .337 \\
MI onset until surgery $<48$ hours & $14(32 \%)$ & $5(21 \%)$ & .335 \\
Posterior septal rupture & $20(46 \%)$ & $13(54 \%)$ & .492 \\
LVEF < 40\% & $14(32 \%)$ & $16(67 \%)$ & .006 \\
Three-vessel disease* & $16(37 \%)$ & $6(27 \%)$ & .423 \\
Complete occlusion of LAD* & $23(54 \%)$ & $7(32 \%)$ & .097 \\
Perioperative variables & & & \\
Urgent operation & $35(80 \%)$ & $23(96 \%)$ & .085 \\
Concomitant CABG & $31(71 \%)$ & $17(71 \%)$ & .974 \\
Greater than 3 bypass grafts & $3(7 \%)$ & $2(8 \%)$ & 1.000 \\
Incomplete revascularization & $5(11 \%)$ & $10(42 \%)$ & .004 \\
Not-revascularized culprit artery & $17(39 \%)$ & $5(23 \%)$ & .178 \\
Concomitant aneurysmectomy & $4(9 \%)$ & $0(0 \%)$ & .289 \\
CPB time $>150$ minutes & $7(16 \%)$ & $8(33 \%)$ & .098 \\
Cardiac arrest time > 100 minutes & $7(16 \%)$ & $5(21 \%)$ & .611 \\
\hline
\end{tabular}

$M I$, Myocardial infarction; $B P$, blood pressure; $I A B P$, intra-aortic balloon pumping; $L V E F$, left ventricular ejection fraction; $L A D$, left anterior descending artery; $C A B G$, coronary artery bypass grafting; $C P B$, cardiopulmonary bypass. *The analysis was performed among the patients who preoperatively underwent coronary angiography ( $\mathrm{n}=43$ in 30-day survivors and $\mathrm{n}=22$ in nonsurvivors).

survived; the other 4 patients died of persistent low cardiac output postoperatively. Preoperative data identifying hemodynamic instability, by low systolic blood pressure, serum creatinine level greater than $250 \mu \mathrm{mol} / \mathrm{L}$, inotrope support, and intra-aortic balloon pump support, were significantly different between 30-day survivors and nonsurvivors. Of note, all 8 patients who had cardiogenic shock, including systolic blood pressure less than $100 \mathrm{~mm} \mathrm{Hg}$ and oliguria with serum creatinine level elevation under inotropic support, died within 30 days postoperatively. Multivariate logistic regression analysis was performed among the patients who preoperatively underwent coronary angiography $(\mathrm{n}=$ 43 in 30-day survivors and $n=22$ in nonsurvivors). Inde- pendent risk factors of 30-day mortality included previous MI $(P=.022)$, previous cardiac surgery $(P=.013)$, preoperative LVEF $<40 \%(P=.046)$, and urgent surgery $(P=$ .016). Incomplete revascularization was not an independent risk factor $(P=.100)$.

\section{Actuarial Survival and Freedom From MACE}

Actuarial survival of all patients was $67 \%$ at 1 year, $63 \%$ at 5 years, $51 \%$ at 10 years, and $36 \%$ at 15 years (Figure $1, A)$. The follow-up period of 30-day survivors was 9.2 \pm 4.9 years with a median being 9.3 years $(0.1-19.7$ years). Among 30-day survivors, 16 (36\%) deaths occurred at 7.8 \pm 4.9 years with a median of 8.9 years $(0.1-17.1$ years $)$ over the study period. Thirteen deaths were confirmed as cardiac death. Of them, 8 deaths were related to congestive cardiac failure. Three patients had out-of-hospital cardiac arrest owing to cardiac arrhythmia suggested by autopsy. Two patients died in the hospital after surgery for residual interventricular communication and LV apex aneurysm, respectively. Three patients died of noncardiac causes. Actuarial survival of 30-day survivors was $95 \%$ at 1 year, $88 \%$ at 5 years, $73 \%$ at 10 years, and $51 \%$ at 15 years (Figure 1, $B)$. MACE included congestive cardiac failure (15 patients), ventricular tachyarrhythmia (6 patients), and redo operation for LV aneurysm (1 patient). No patients had acute coronary syndrome during the follow-up. Actuarial freedom from MACE of 30-day survivors was $91 \%$ at 1 year, $61 \%$ at 5 years, $40 \%$ at 10 years, and $19 \%$ at 15 years (Figure 1, $C$ ).

\section{Congestive Cardiac Failure}

A total of $20(45.5 \%)$ patients had congestive cardiac failure at $5.4 \pm 4.4$ years with a median of 3.9 years $(0.1-15.2$ years). Among them, 7 patients were related to residual interventricular communication and underwent surgical repair. One patient had severe ischemic mitral regurgitation and underwent mitral valve replacement. Actuarial freedom from congestive cardiac failure of 30-day survivors was $93 \%$ at 1 year, $70 \%$ at 5 years, $54 \%$ at 10 years, and $28 \%$ at 15 years (Figure 2, $A$ ). The Cox proportional hazards model identified history of hypertension, posterior septal rupture, residual interventricular communication, and preoperative LVEF less than $40 \%$ as the predictors of congestive cardiac failure over the long term (Table 2).

\section{Ventricular Tachyarrhythmia}

Twelve $(27 \%)$ patients had ventricular tachyarrhythmia treated in the hospital at $6.2 \pm 4.0$ years with a median 5.9 years (0.4-13.0 years). Among them, 8 patients received an automatic implantable cardiac defibrillator, whereas 4 patients who presented after 1 or 2 episodes of nonsustained ventricular tachycardia with preserved LV function were treated medically. Actuarial freedom from ventricular tachyarrhythmia of 30 -day survivors was $98 \%$ at 1 year, $85 \%$ at 5 

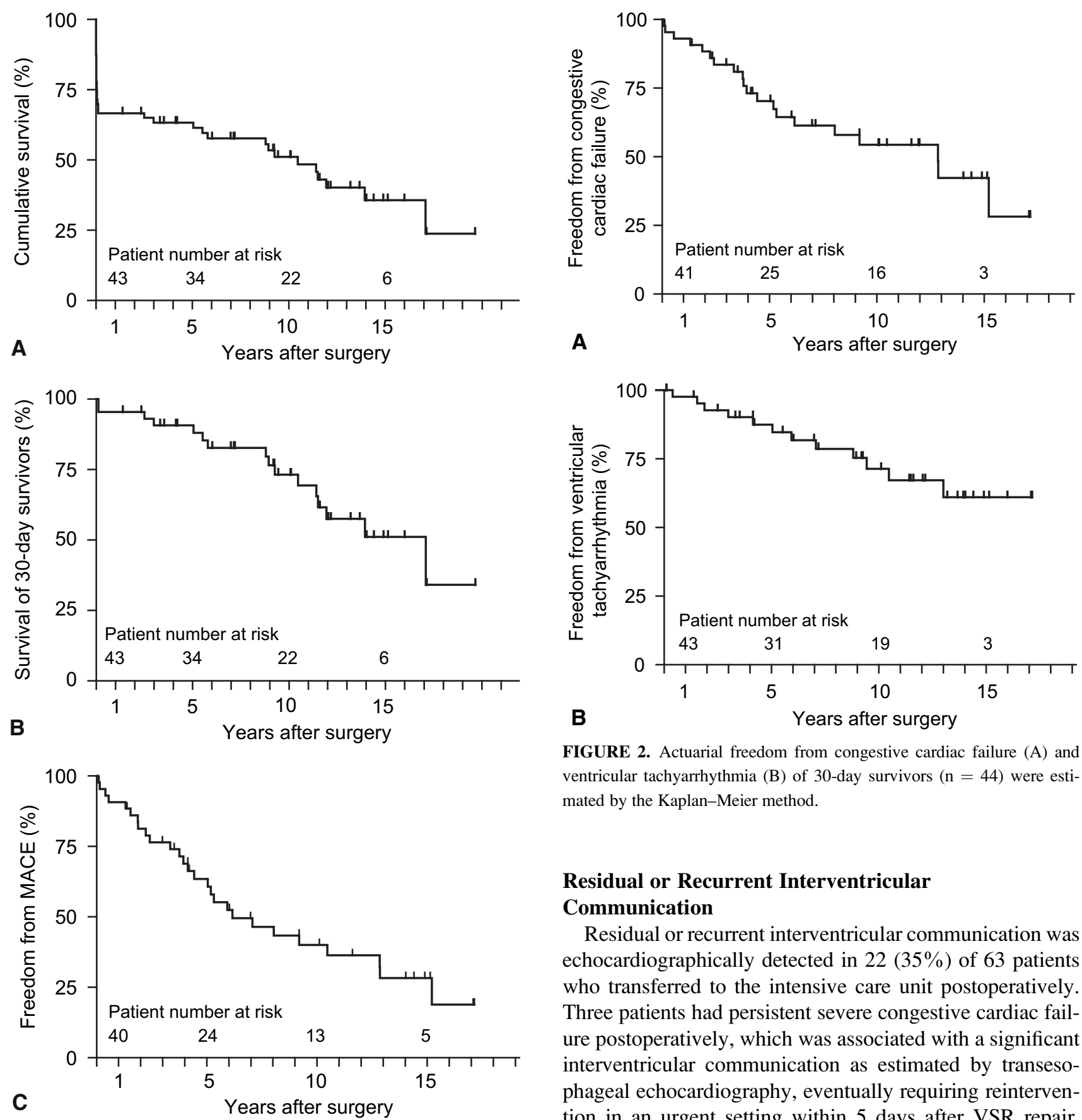

FIGURE 2. Actuarial freedom from congestive cardiac failure (A) and ventricular tachyarrhythmia $(B)$ of 30-day survivors $(n=44)$ were estimated by the Kaplan-Meier method.

\section{Residual or Recurrent Interventricular Communication}

Residual or recurrent interventricular communication was echocardiographically detected in $22(35 \%)$ of 63 patients who transferred to the intensive care unit postoperatively. Three patients had persistent severe congestive cardiac failure postoperatively, which was associated with a significant interventricular communication as estimated by transesophageal echocardiography, eventually requiring reintervention in an urgent setting within 5 days after VSR repair. Among them, surgical repair was performed in 2 patients, who were discharged from the hospital without further morbidities, whereas percutaneous repair was successfully performed using an Amplatzer septal occluder ${ }^{20}$ in 1 patient, who later died of anemia that could not be treated because of the patient's religious convictions. Six patients underwent surgical repair of residual/recurrent communication for refractory congestive cardiac failure associated with a significant interventricular communication in a semiurgent/ elective setting between 24 and 202 days after VSR repair.

years, $71 \%$ at 10 years, and $61 \%$ at 15 years (Figure $2, B$ ). The Cox proportional hazards model identified concomitant $\mathrm{LV}$ aneurysmectomy and complete occlusion of the LAD as significant independent predictors of ventricular tachyarrhythmia over the long term (Table 3).
Of them, 1 patient died in the operating room of global 
TABLE 2. Independent predictors of congestive cardiac failure

\begin{tabular}{lccc}
\hline \multicolumn{1}{c}{ Predictors } & Odds ratio & $\mathbf{9 5} \%$ CI & $\boldsymbol{P}$ value \\
\hline History of hypertension & 6.14 & $1.72-21.9$ & .005 \\
$\begin{array}{l}\text { Diabetes } \\
\text { Preoperative serum }\end{array} \quad 0.35$ & $0.03-3.62$ & .377 \\
$\quad$ creatinine $>250 \mu \mathrm{mol} / \mathrm{L}$ & 4.62 & $0.52-40.8$ & .169 \\
MI onset & & & \\
$\quad$ until surgery $<48$ hours & 2.44 & $0.68-8.83$ & .173 \\
Posterior septal rupture & & & \\
Preoperative LVED $<40 \%$ & 4.44 & $1.24-15.8$ & .022 \\
Three-vessel disease & 3.86 & $1.33-11.2$ & .013 \\
Residual interventricular & 0.75 & $0.23-2.39$ & .620 \\
$\quad$ communicaiton & 4.41 & $1.22-15.9$ & .024 \\
CI, Confidence interval; $M I$, myocardial infarction; $L V E F$, left ventricular ejection \\
fraction.
\end{tabular}

myocardial dysfunction, whereas the other 5 patients survived until the completion of follow-up of this study without further MACE. Two patients successfully underwent surgical repair of residual/recurrent interventricular communication at 2 years and 10 years after VSR repair without further MACE. Dehiscence between the patch and infarcted myocardium produced an interventricular communication in all patients who underwent surgical repair of residual communication. The other 11 patients who did not undergo repair of residual communication until the completion of this study had been asymptomatic with persisting minimal communication (echocardiographic pulmonary/systemic flow ratio $<1.5$ ) and preserved LV function.

\section{DISCUSSION}

We here demonstrate 30-day and long-term (mean followup period of 9.2 years) clinical outcomes of 68 patients who underwent surgical repair of postinfarction VSR in a single institution that performed more than 35,000 open cardiac operations between 1988 and 2007. Thirty-day mortality was $35 \%$, with previous MI, previous cardiac surgery, preoperative LVEF less than $40 \%$, and urgent surgery being independent risk factors. Residual interventricular commu-

TABLE 3. Independent predictors of ventricular tachyarrhythmia

\begin{tabular}{lccc}
\hline \multicolumn{1}{c}{ Predictors } & Odds ratio & $\mathbf{9 5 \%}$ CI & $\boldsymbol{P}$ value \\
\hline History of hypertension & 0.13 & $0.01-1.25$ & .077 \\
Previous MI & 2.98 & $0.33-26.8$ & .329 \\
$\begin{array}{l}\text { Preoperative serum } \\
\quad \text { creatinine }>250 \mu \mathrm{mol} / \mathrm{L}\end{array}$ & 9.66 & $0.77-122$ & .079 \\
MI onset & & & \\
$\quad$ until surgery $<48$ hours & 3.06 & $0.58-16.2$ & .189 \\
Preoperative LVEF $<40 \%$ & & & \\
Three-vessel disease & 1.97 & $0.52-7.51$ & .318 \\
Occlusion of LAD & 0.41 & $0.09-1.94$ & .258 \\
Concomitant LV aneurysmectomy & 14.80 & $1.64-134$ & .016 \\
\hline
\end{tabular}

$C I$, Confidence interval; $M I$, myocardial infarction; $L V E F$, left ventricular ejection fraction; $L A D$, left anterior descending artery; $L V$, left ventricle. nication was echocardiographically detected in 22 patients and 11 patients underwent reintervention. Actuarial survival and freedom from MACE of 30-day survivors were $88 \%$ and $61 \%$ at 5 years, $73 \%$ and $40 \%$ at 10 years, and $51 \%$ and $19 \%$ at 15 years, respectively. Actuarial freedom from congestive cardiac failure and ventricular tachyarrhythmia of 30-day survivors were $70 \%$ and $85 \%$ at 5 years, $54 \%$ and $71 \%$ at 10 years, and $28 \%$ and $61 \%$ at 15 years, respectively. Interestingly, independent predictors were different between congestive cardiac failure and ventricular tachyarrhythmia. Independent predictors for congestive cardiac failure included history of hypertension, posterior septal rupture, residual interventricular communication, and preoperative LVEF less than $40 \%$, whereas concomitant LV aneurysmectomy and complete occlusion of the LAD were independent predictors of ventricular tachyarrhythmia.

Thirty-day mortality after repair of postinfarction VSR in this study (35\%) was comparable with the majority of previous reports. ${ }^{1,3,6,7,9,11-14,17}$ Consistent with previous reports, ${ }^{3,6,7,9,13}$ all patients who had cardiogenic shock died within 30 days, suggesting that prompt diagnosis and assessment followed by urgent surgical intervention before hemodynamic deterioration would be particularly important to improve in-hospital outcomes. In addition, preoperative poor $\mathrm{LV}$ function was an independent risk factor, suggesting that procedures to improve LV contractility, such as CABG, might be important to improve outcomes, although incomplete revascularization was not an independent risk factor in this study. Urgent surgery was an independent predictor for 30-day mortality in this study. However, this finding not only suggests that urgent surgery elevates the risk of in-hospital mortality, but also indicates that mortality of elective intervention would be very low. Previous reports have suggested that posterior septal rupture is a risk factor of 30-day mortality owing to its more complex pathology than anterior septal rupture, including involvement of the subvalvular apparatus and right ventricular function. ${ }^{11,12}$ This study, however, does not indicate posterior septal rupture as a risk factor. Extensive exposure of infarct area via ventriculotomy and/or atrioventricular valves to securely repair the ruptured septum might minimize residual interventricular communication and alteration in ventricular geometries, consequently improving in-hospital outcomes. A short interval between MI onset and surgical intervention has also been reported to be a risk factor of 30-day mortality, suggesting that early presentation of VSR represents extensive myocardial necrosis, leading to poor outcomes. ${ }^{2,9,12,17}$ This study, however, indicates that MI onset until VSR repair of less than 48 hours is not a risk factor of 30-day mortality. Such a discrepancy could be explained by a difference in management strategy. ${ }^{7}$ In our strategy, surgery was carried out urgently after the diagnosis of VSR, whereas Blanche, ${ }^{17}$ Jones, ${ }^{12}$ and their associates delayed the surgical repair of VSR unless hemodynamics was compromised. ${ }^{12,17}$ 
Preoperative coronary angiography has been routinely performed in patients with a diagnosis of postinfarction VSR in our institution in the past 20 years. Recently, VSR associated with multivessel coronary artery disease is reported to be more prevalent than that associated with occlusion of a single major coronary artery with other arteries being intact, which was common in the prethrombolysis/reperfusion era. ${ }^{2}$ In our series, there were no differences in coronary artery pathologic conditions over the past 20 years, with $69 \%$ of patients having multivessel coronary artery disease. As a result, concomitant CABG was performed in $71 \%$ of total VSR patients. This finding suggests that coronary angiography is a very important preoperative diagnostic tool in postinfarct VSR to possibly improve in-hospital and long-term surgical outcomes with sufficient supporting evidence. $^{6,7,14,15}$

Residual interventricular communication was detected in $22(32 \%)$ patients. Of them, $11(50 \%)$ underwent intervention for residual communication, whereas the other 11 patients were asymptomatic with a small residual communication. It is important to carefully define the suture line of the patch to repair the VSR intraoperatively, inasmuch as progressive necrosis of myocardium near the suture line leads to dehiscence, as seen in our series and previous reports. ${ }^{21}$ Although delayed repair might allow secure repair to prevent residual/recurrent interventricular communication, ${ }^{12,17}$ it also carries a substantial risk of hemodynamic deterioration. ${ }^{4}$ Most important, prompt and proper diagnosis and management of residual interventricular communication are essential to improve clinical outcomes. In the early period after VSR repair, transesophageal echocardiography was particularly useful in diagnosing the cause of cardiac failure among a variety of possible causes. We successfully diagnosed and repaired residual communication in 3 patients within 5 days after VSR repair. Moreover, residual communication was the leading predictor of congestive cardiac failure long term. We performed surgical intervention in 6 patients within 1 year and 2 patients in a later period after VSR repair with 1 in-hospital mortality, illustrating that close follow-up is required for patients having residual communication. Although surgery is the standard strategy for intervention of residual communication, percutaneous device closure is also promising. Although percutaneous closure of postinfarction VSR has been suggested to carry technical difficulties, such as placement of the device without distorting ventricular geometry or causing atrioventricular regurgitation, ${ }^{2}$ tissues where the device is placed in residual communication might be more supportive compared with necrotic myocardium in postinfarction VSR, as we successfully performed in 1 patient 5 days after VSR repair.

This study demonstrates long-term outcomes for postinfarction VSR with a mean follow-up being 9.2 years. Fiveand 10-year actuarial survival of 30-day survivors in this study $(88 \%$ and $73 \%$, respectively) was consistent with the majority of previous studies. ${ }^{7-9,11-14,16}$ Although 2 recently published reports ${ }^{6,7}$ demonstrated long-term survival after VSR repair and Lundblad and associates ${ }^{7}$ identified independent risk factors of long-term mortality, incidences of MACE or factors affecting MACE after VSR repair are still poorly understood. ${ }^{11,16}$ We here clearly demonstrate substantial incidences of MACE and independent risk factors of congestive cardiac failure and ventricular tachyarrhythmia.

Congestive cardiac failure occurred in $46 \%$ of 30-day survivors. History of hypertension and residual interventricular communication were leading predictors of long-term congestive cardiac failure. Other predictors included preoperative LVEF less than $40 \%$, which might be due to pre-existing cardiac disease or necrotic/hibernating LV myocardium. Interestingly, posterior septal rupture was also an independent predictor of congestive cardiac failure over the long term. More complex pathologic conditions in posterior rupture than anterior rupture might affect longterm systolic and diastolic ventricular function. In addition, patch repair of posterior VSR might alter geometry of the base of the ventricular myocardium and/or affect the subvalvular apparatus, resulting in ventricular dysfunction. These findings suggest that intensive medical treatment by angiotensin-converting enzyme inhibitor, $\beta$-blocker, or aldosterone blocker might need to be commenced in the early period depending on background and perioperative variables.

In this study, $27 \%$ of 30 -day survivors had ventricular tachyarrhythmia, whereas Davies and colleagues ${ }^{16}$ identified that $40 \%$ of patients showed frequent ventricular premature beats and only $5 \%$ of 43 patients had ventricular tachycardia after VSR repair. This discrepancy might be explained by difference in follow-up period (median follow-up of 114 months in this study vs 30 months in Davies' study $\left.{ }^{16}\right)$. Concomitant LV aneurysmectomy and occlusion of the LAD were predictors of ventricular tachyarrhythmia long term, suggesting that extensive myocardial infarction followed by full-thickness scar formation at the ventricle would be the most important factor to induce ventricular tachyarrhythmia.

Some limitations are due to the retrospective nature. First, preoperative right ventricular function, which has been suggested to be a risk factor of in-hospital mortality, $8,12,13,19$ was not included in the analysis, inasmuch as this parameter was not consistently measured throughout the study period. Second, surgical procedures, including myocardial protection, approach, patch placement technique, or patch materials, were slightly different over the study period and operating surgeons. Third, medical treatment long term varied, although all the patients were treated by specialist surgeons or physicians. Finally, this study could not identify any differences in 30-day or long-term outcomes over the study period, despite substantial changes in perioperative managements. Considering high in-hospital mortality, 
randomized controlled trials for perioperative management would be difficult. Therefore, prospective collection of data in a multicenter basis is needed to verify the results of this study and to further improve in-hospital and long-term surgical outcomes of postinfarction VSR.

In conclusion, prompt management is particularly important to improve in-hospital and long-term outcomes for postinfarction VSR. Long-term outcomes after surgical repair of post-MI VSR were favorable, despite infrequent exposure by individual surgeons to the pathologic conditions, indicating that aggressive surgical approach is warranted. As predictors of congestive cardiac failure and ventricular arrhythmia varied over the long term, intensive medical treatment depending on background or perioperative variables might be effective to improve long-term outcomes.

We acknowledge Mrs Susan Smith, Miss Lesley Drake, Miss Julie-Gai Harris, and Mrs Joanne McNamara at Cardiac Surgical Clinical Information Service in The Prince Charles Hospital for data collection and management.

\section{References}

1. Crenshaw BS, Granger CB, Birnbaum Y, Pieper KS, Morris DC, Kleiman NS, et al. Risk factors, angiographic patterns, and outcomes in patients with ventricular septal defect complicating acute myocardial infarction. GUSTO-I (Global Utilization of Streptokinase and TPA for Occluded Coronary Arteries) Trial Investigators. Circulation. 2000;101:27-32.

2. Birnbaum Y, Fishbein MC, Blanche C, Siegel RJ. Ventricular septal rupture after acute myocardial infarction. $N$ Engl J Med. 2002;347:1426-32.

3. Poulsen SH, Praestholm M, Munk K, Wierup P, Egeblad H, Nielsen-Kudsk JE. Ventricular septal rupture complicating acute myocardial infarction: clinical characteristics and contemporary outcome. Ann Thorac Surg. 2008;85:1591-6.

4. Ryan TJ, Antman EM, Brooks NH, Califf RM, Hillis LD, Hiratzka LF, et al. 1999 update: ACC/AHA guidelines for the management of patients with acute myocardial infarction. A report of the American College of Cardiology/American Heart Association Task Force on Practice Guidelines (Committee on Management of Acute Myocardial Infarction). J Am Coll Cardiol. 1999;34:890-911.

5. Mehta RH, Grab JD, O'Brien SM, Glower DD, Haan CK, Gammie JS, et al. Clinical characteristics and in-hospital outcomes of patients with cardiogenic shock undergoing coronary artery bypass surgery: insights from the Society of Thoracic Surgeons National Cardiac Database. Circulation. 2008;117:876-85.
6. Papadopoulos N, Moritz A, Dzemali O, Zierer A, Rouhollapour A, Ackermann H, et al. Long-term results after surgical repair of postinfarction ventricular septal rupture by infarct exclusion technique. Ann Thorac Surg. 2009;87:1421-5.

7. Lundblad R, Abdelnoor M, Geiran OR, Svennevig JL. Surgical repair of postinfarction ventricular septal rupture: risk factors of early and late death. $J$ Thorac Cardiovasc Surg. 2009;137:862-8.

8. Blanche C, Khan SS, Chaux A, Matloff JM. Postinfarction ventricular septal defect in the elderly: analysis and results. Ann Thorac Surg. 1994;57:1244-7.

9. Deja MA, Szostek J, Widenka K, Szafron B, Spyt TJ, Hickey MS, et al. Post infarction ventricular septal defect-can we do better? Eur J Cardiothorac Surg. 2000;18:194-201.

10. Deville C, Fontan F, Chevalier JM, Madonna F, Ebner A, Besse P. Surgery of post-infarction ventricular septal defect: risk factors for hospital death and longterm results. Eur J Cardiothorac Surg. 1991;5:167-74.

11. Jeppsson A, Liden H, Johnsson P, Hartford M, Radegran K. Surgical repair of post infarction ventricular septal defects: a national experience. Eur J Cardiothorac Surg. 2005;27:216-21.

12. Jones MT, Schofield PM, Dark JF, Moussalli H, Deiraniya AK, Lawson RA, et al Surgical repair of acquired ventricular septal defect: determinants of early and late outcome. J Thorac Cardiovasc Surg. 1987;93:680-6.

13. Labrousse L, Choukroun E, Chevalier JM, Madonna F, Robertie F, Merlico F, et al. Surgery for post infarction ventricular septal defect (VSD): risk factors for hospital death and long term results. Eur J Cardiothorac Surg. 2002;21:725-31.

14. Pretre R, Ye Q, Grunenfelder J, Zund G, Turina MI. Role of myocardial revascularization in postinfarction ventricular septal rupture. Ann Thorac Surg. 2000;69: 51-5.

15. Dalrymple-Hay MJ, Langley SM, Sami SA, Haw M, Allen SM, Livesey SA, et al. Should coronary artery bypass grafting be performed at the same time as repair of a post-infarct ventricular septal defect? Eur J Cardiothorac Surg. 1998;13:286-92.

16. Davies RH, Dawkins KD, Skillington PD, Lewington V, Monro JL, Lamb RK, et al. Late functional results after surgical closure of acquired ventricular septal defect. J Thorac Cardiovasc Surg. 1993;106:592-8.

17. Blanche C, Khan SS, Matloff JM, Chaux A, DeRobertis MA, Czer LS, et al. Results of early repair of ventricular septal defect after an acute myocardial infarction. J Thorac Cardiovasc Surg. 1992;104:961-5.

18. Daggett WM, Guyton RA, Mundth ED, Buckley MJ, McEnany MT, Gold HK, et al. Surgery for post-myocardial infarct ventricular septal defect. Ann Surg. 1977; 186:260-71.

19. David TE, Dale L, Sun Z. Postinfarction ventricular septal rupture: repair by endocardial patch with infarct exclusion. J Thorac Cardiovasc Surg. 1995;110: 1315-22.

20. Lee EM, Roberts DH, Walsh KP. Transcatheter closure of a residual postmyocardial infarction ventricular septal defect with the Amplatzer septal occluder. Heart. 1998;80:522-4.

21. Skillington PD, Davies RH, Luff AJ, Williams JD, Dawkins KD, Conway N, et al Surgical treatment for infarct-related ventricular septal defects: improved early results combined with analysis of late functional status. J Thorac Cardiovasc Surg. 1990;99:798-808. 